\title{
First use of a scoring balloon to perform Oddi sphincteroplasty for biliary stones
}

\author{
Andrea Coppola ${ }^{a}$, Filippo Piacentino ${ }^{a}$, Edoardo Macchi ${ }^{a}$, Giuseppe De Marchia , Anna Maria lerardi ${ }^{b}$, \\ Gianpaolo Carrafiello ${ }^{b}$, Federico Fontana ${ }^{a}$
}

ASST Sette Laghi, University of Insubria, Varese; San Paolo Hospital, University of Milan, Italy

\begin{abstract}
Balloon sphincteroplasty is a well-known procedure for the treatment of biliary stones. Scoring balloons are characterized by rigid tangential elements that provide focal force, achieving a greater pressure than normal balloons. We report the first (to our knowledge) use of a scoring balloon to perform sphincteroplasty in 4 cases of elderly people presenting with symptomatic choledocholithiasis. In all cases we achieved complete common bile duct clearing with good symptom relief. No procedural complications were observed.
\end{abstract}

Keywords Gallstones, calculi, cholangiography, transhepatic sphincteroplasty, sphincter of Oddi, interventional radiology

Ann Gastroenterol 2019; 32 (4): 1-3

\section{Introduction}

Oddi's balloon sphincteroplasty is nowadays a well-known procedure for the treatment of choledocholithiasis, safer than sphincterotomy and similarly effective $[1,2]$. Both retrograde and antegrade approaches are described and suggested by current guidelines [3]. Scoring balloons are used in endovascular procedures to achieve a higher dilating force [4-6]; however, to our knowledge, they had never been used in the biliary procedure setting. The purpose of this study was to evaluate the feasibility of scoring balloon-mediated percutaneous Oddi sphincteroplasty.

\section{Patients and methods}

\section{Patients}

From September 2017 to June 2018, 4 patients (2 men and 2 women), mean age 82 (range 70-89) years, presented to our institution with symptomatic choledocholithiasis.

Department of Diagnostic and Interventional Radiology, ${ }^{a}$ ASST Sette Laghi, University of Insubria, Varese (Andrea Coppola, Filippo Piacentino, Edoardo Macchi, Giuseppe De Marchi, Federico Fontana); bSan Paolo Hospital, University of Milan (Anna Maria Ierardi, Gianpaolo Carrafiello), Italy

Conflict of Interest: None

Correspondence to: Andrea Coppola, Radiology Department, ASST Sette Laghi, Ospedale di Circolo e Fondazione Macchi, Via Guicciardini 9, Varese 21100, Italy, e-mail: coppola.andrea.mg@gmail.com

Received 16 December 2018; accepted 3 April 2019; published online 25 April 2019

DOI: https://doi.org/10.20524/aog.2019.0380
Two of the 4 cases were complicated by cholecystitis, another by Clostridium difficile (C. difficile) enteric infection. All patients underwent ultrasound and magnetic resonance cholangiopancreatography, which revealed the migration of one or more gallstones to the main bile duct (Fig. 1).

Endoscopic retrograde cholangiopancreatography (ERCP) was attempted in 2 of the cases, but unsuccessfully. In one case it was not possible to pass the guidewire through the Vater papilla into the common bile duct; in the second, the Vater papilla could not be reached because of a surgically altered anatomy (gastric resection for a duodenal ulcer 50 years before). In the remaining 2 cases, ERCP could not be performed because of previous surgery (gastric bypass for obesity and total gastrectomy for adenocarcinoma).

\section{Procedure}

All procedures were carried out under moderate sedation and analgesia (appropriate doses of propofol, fentanyl and midazolam under anesthesiologist's supervision) and with appropriate antibiotic prophylaxis (single administration of ciprofloxacin $1 \mathrm{~g}$, immediately before the procedure).

All procedures were performed in the angiography suite under fluoroscopic guidance (AlluraXper FD20, Philips Healthcare, Best, The Netherlands). In all patients, percutaneous transhepatic cholangiography was performed using a 21-22-G Chiba needle (Chibell, BPB, Modena, Italy) to opacify the biliary system and confirm choledocholithiasis (Fig. 2A); a peripheral bile duct was catheterized with an 18-G Ring needle cannula (Drainage Access Catheter, Cook, Bloomington, IN, USA). We used a guidewire catheter support (0.035", Glidewire, Terumo, Tokyo, Japan; 5 Fr, 


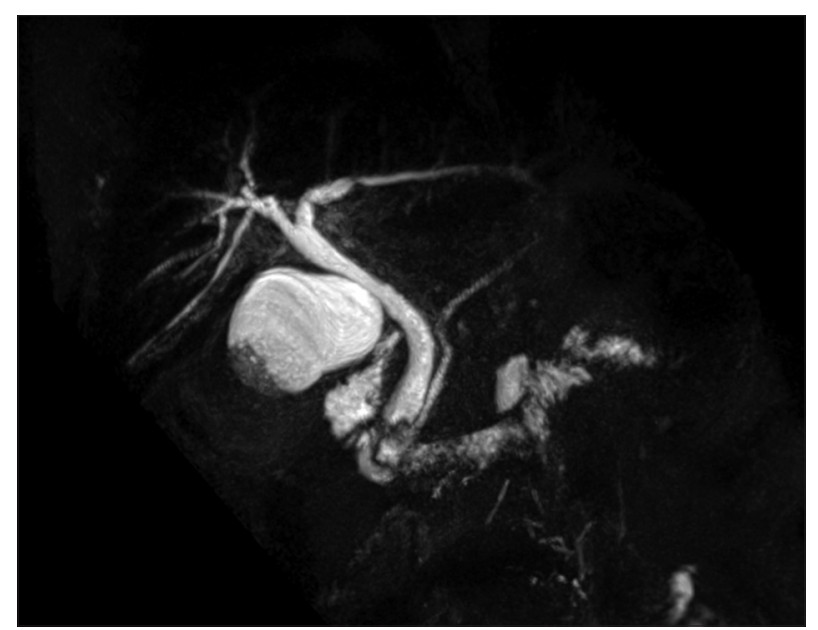

Figure 1 Pre-proceduralmagneticresonancecholangiopancreatography demonstrating a signal void in the main bile duct, attributable to a biliary stone
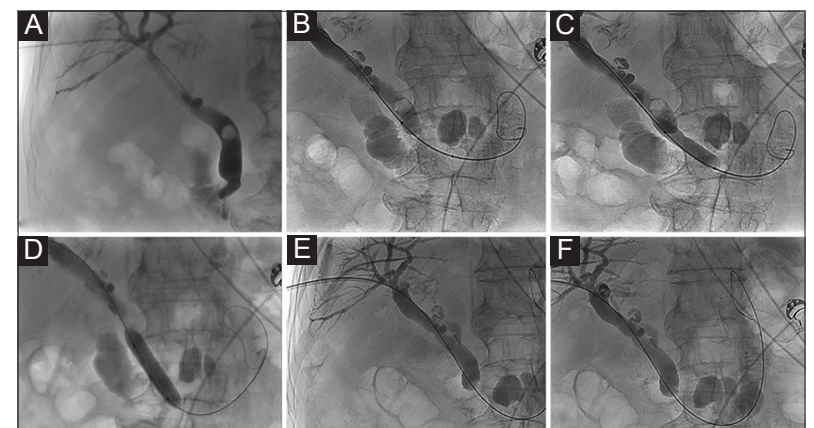

Figure 2 Percutaneous transhepatic cholangiography (PTC) demonstrating a round-shaped defect in the main bile duct, attributable to a biliary stone (A). The Advance Enforcer percutaneous transluminal angioplasty balloon catheter is advanced (B) and then inflated (C) at the level of Oddi's sphincter, achieving sphincteroplasty. A standard occlusion balloon is used to gently push the stones through the sphincteroplasty into the duodenum (D). Confirmatory PTC demonstrated complete clearing of stones from the main bile duct (E, F)

Cordis, Miami, FL, USA) to cross the occlusion. An 8-Fr vascular introducer sheath (Cordis, Baar, Switzerland) was then positioned on a 0.035 " stiff guidewire (Amplatz, Boston Scientific, Ratingen, Germany) with the distal tip at the ligament of Treitz.

In an off-label setting, we introduced a $10 \mathrm{~mm}$ diameter, $40 \mathrm{~mm}$ length Advance Enforcer 35 Focal-Force percutaneous transluminal angioplasty (PTA) balloon catheter (Cook, Bloomington, IN, USA) over the stiff guidewire. This was inflated at the level of Oddi's sphincter (Fig. 2B-C) to achieve the sphincteroplasty.

The Enforcer balloon was then removed and a standard occlusion balloon catheter (Powerflex Pro 0.035" PTA Catheter, Cordis, Baar, Switzerland) was advanced over the stiff guidewire, inflated proximally to the obstruction, and used to gently push the stones through the sphincteroplasty into the duodenum (Fig. 2D).

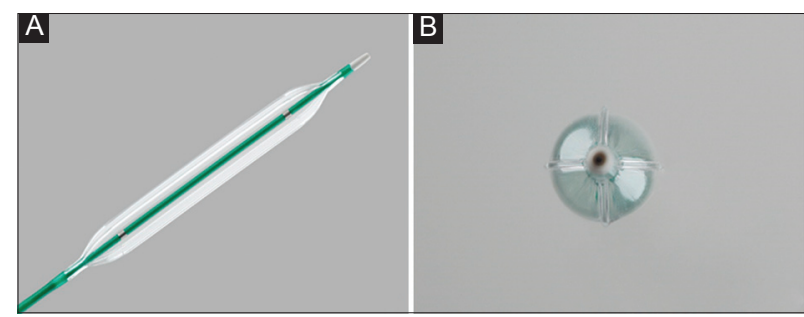

Figure 3 (A) The Advance Enforcer (Cook, Bloomington, IN, USA) scoring balloon for percutaneous transluminal angioplasty. (B) Note the 4 metal-free orthogonally arranged rigid polymer elements that provide focal force to obtain high-pressure dilatation

At the end of the procedure (Fig. 2E-F) and 7 days later, confirmatory percutaneous transhepatic cholangiography and a cone-beam computed tomography scan with biliary administration of contrast media (cholangio-CBCT) were used to check for complete clearance of the main bile duct. After the 7 -day check, the $8.5 \mathrm{~F}$ internal-external biliary drainage was removed.

Mean overall procedure time was 27.5 (range 10-40) min, measured as the time from the placement of the patient on the angiographic bed until the end of the procedure (patient's exit from the angiography suite). The mean radiation dose of the procedure in terms of dose-area product was 53.12 (range 30.44-78.06) Gy.cm².

\section{Balloon description}

The Advance Enforcer (Cook, Bloomington, IN, USA) is a dual lumen shaft PTA scoring balloon catheter, characterized by 4 orthogonally arranged rigid polymer elements that provide focal force (Fig. 3). The rigid polymer elements are metal-free and can generate a rated burst pressure up to 11 atmospheres (atm). It is designed for PTA of peripheral arteries, as well as for obstructive lesions of native or synthetic arteriovenous dialysis fistulas [7].

\section{Results}

No intraprocedural complications were recorded, according to the Common Terminology Criteria for Adverse Events (CTCAE), Version 5.0 [8]. Technical success, defined as complete clearing of gallstones from the common bile ducts, was achieved in all patients. Clinical success, defined as clinical relief, was completely obtained in 3 of the 4 patients and partially obtained in one.

\section{Discussion}

Balloon sphincteroplasty is a fast, safe and useful solution for treating biliary stones [1,2,9]. Scoring balloons are 
characterized by rigid elements (polymer or metallic) that provide focal radial force along the edges of the scoring element when the balloon is inflated. They have been used safely for many vascular procedures and they achieve better luminal expansion compared to conventional balloon angioplasty, especially in complex fibro-calcific lesions, resulting in reduced barotrauma and less elastic recoil, with a lower incidence of vascular dissections [4-6].

Despite the fact that the fibrosis at the bottom of Oddi's sphincter stricture and the atherosclerotic plaque are of a different nature, we hypothesized that a scoring balloon could achieve better results compared with the conventional balloon technique (a sphincterotomy-like sphincteroplasty) reported in the endovascular literature [4-6]. We choose to use a metalfree scoring balloon to avoid excessive radial force and prevent lesions of the biliary duct wall.

Our preliminary results appear to be promising: we obtained an excellent technical success rate, with complete clearing of the common bile duct in all patients. Clinical success was complete in only 3 of 4 patients: one patient reported only partial symptom relief, but she also suffered from $C$. difficile enteric infection.

In older patients who present with symptomatic biliary stones, the endoscopic retrograde cholangiography approach may be arduous because of tissue laxity or surgically altered anatomy, as in the cases reported here. In these patients, interventional radiology may offer assistance in the form of either laser lithotripsy [10] or balloon sphincteroplasty. Several studies with a larger numbers of patients and a longer follow up are needed to assess the safety and overall outcomes of this procedure.

\section{References}

1. Turner GA, Ing AJ, Connor SJ. Endoscopic large balloon sphincteroplasty is a useful, safe adjunct for difficult to treat choledocholithiasis. ANZ J Surg 2016;86:395-398.

2. Szulman C, Giménez M, Sierre S. Antegrade papillary balloon dilation for extrahepatic bile duct stone clearance: lessons learned from treating 300 patients. J Vasc Interv Radiol 2011;22:346-353.

3. Testoni PA, Mariani A, Aabakken L, et al. Papillary cannulation and sphincterotomy techniques at ERCP: European Society of Gastrointestinal Endoscopy (ESGE) Clinical Guideline. Endoscopy 2016;48:657-683.

4. Scheinert D, Peeters P, Bosiers M, O’Sullivan G, Sultan S, Gershony G. Results of the multicenter first-in-man study of a novel scoring balloon catheter for the treatment of infra-popliteal peripheral arterial disease. Catheter Cardiovasc Interv 2007;70:1034-1039.

5. Bosiers M, Deloose K, Cagiannos C, Verbist J, Peeters P. Use of the AngioSculpt scoring balloon for infrapopliteal lesions in patients with critical limb ischemia: 1-year outcome. Vascular 2009;17:29-35.

6. Fonseca A, Costa JR Jr, Abizaid A, et al. Intravascular ultrasound assessment of the novel AngioSculpt scoring balloon catheter for the treatment of complex coronary lesions. J Invasive Cardiol 2008;20:21-27.

7. Cook Medical. Advance ${ }^{\circledR}$ Enforcer $^{\mathrm{TM}} 35$ Focal-Force PTA Balloon Catheter. https://www.cookmedical.eu/products/85776322-9c38-4ff59aaa-8143d2e7b8a8 [Accessed 15 April 2019].

8. National Cancer Institute (U.S.). Common terminology criteria for adverse events (CTCAE). Version 5.0. 2017.

9. Liao WC, Tu YK, Wu MS, et al. Balloon dilation with adequate duration is safer than sphincterotomy for extracting bile duct stones: a systematic review and meta-analyses. Clin Gastroenterol Hepatol 2012;10:1101-1109.

10. Ierardi AM, Fontana F, Petrillo M, et al. Percutaneous transhepatic endoscopic holmium laser lithotripsy for intrahepatic and choledochal biliary stones. Int J Surg 2013;11 Suppl 1:S36-S39. 